\title{
High-Power Hybrid Mode-Locked External Cavity Semiconductor Laser Using Tapered Amplifier with Large Tunability
}

\author{
Andreas Schmitt-Sody, Andreas Velten, Ye Liu, Ladan Arissian, and Jean-Claude Diels \\ Department of Physics and Astronomy and Center for High Technology Materials, University of New Mexico, \\ Albuquerque, NM 87106, USA \\ Correspondence should be addressed to Jean-Claude Diels, jcdiels@unm.edu
}

Received 4 April 2008; Accepted 17 June 2008

Recommended by John McInerney

We report on hybrid mode-locked laser operation of a tapered semiconductor amplifier in an external ring cavity, generating pulses as short as $0.5 \mathrm{ps}$ at $88.1 \mathrm{MHz}$ with an average power of $60 \mathrm{~mW}$. The mode locking is achieved through a combination of a multiple quantum well saturable absorber ( $>10 \%$ modulation depth) and an RF current modulation. This designed laser has $20 \mathrm{~nm}$ tuning bandwidth in continuous wave and $10 \mathrm{~nm}$ tuning bandwidth in mode locking around $786 \mathrm{~nm}$ center wavelength at constant temperature.

Copyright (C) 2008 Andreas Schmitt-Sody et al. This is an open access article distributed under the Creative Commons Attribution License, which permits unrestricted use, distribution, and reproduction in any medium, provided the original work is properly cited.

\section{Introduction}

Ultrashort-pulse semiconductor lasers have been introduced as compact, efficient, low-cost sources for a wide variety of applications, ranging from nonlinear frequency conversion, remote sensing, and spectroscopy, to free-space communication. Pioneering work with mode-locked semiconductor lasers dates back to the early 1980's [1,2]. Despite two decades of extensive research on these lasers, their performances have not yet approached those of crystalline host laser systems in terms of high output power and short pulse duration. One of the basic challenges is that the small cross-section area of the standard narrow stripe semiconductor laser limits the power that can be extracted before laser damage occurs. Therefore the average output power is limited to only a couple of milliwatts, and the energy typically to $20 \mathrm{pJ}$ per pulse [3]. Another difficulty is the complex nonlinear interrelation between the optical field, the current density, and the index of refraction, resulting in pulse-phase modulation that can not be easily compensated. Pulse durations range from a few picoseconds to just under one picosecond, employing intracavity dispersion compensation. There are two possible solutions to generate high average power short pulses with semiconductor lasers without any external amplification elements: (i) use of high average power vertical-external-cavity surface-emitting (VECSEL) semiconductor lasers in ring or linear cavities [4] and (ii) use of tap amplifiers (TAs) in a ring oscillator configuration which is the approach taken in this paper. Tapered semiconductor amplifiers are laser diodes with an additional guiding structure confining the beam in the plane of the semiconductor junction $[5,6]$. The basic goal is to avoid saturation and damage due to high intensities by having the beam expanded throughout its propagation into the semiconductor material. A variant commonly used today has an index guided portion for initial amplification, followed by a gain guided expanding (tapered) region. After leaving the initial waveguide the beam diffracts practically freely in the dimension along the semiconductor junction. In the plane perpendicular to the junction it stays confined until it leaves the semiconductor. Using two cylindrical lenses, it is then possible to create a high-quality, near circular, collimated, high-power output beam. Tapered amplifiers are more commonly used in a master-oscillator power amplifier (MOPA) setup, where the temporal and spatial characteristics of the output of a low-power oscillator are well maintained through amplification in a tapered structure $[7,8]$. Instead, in the configuration presented here, the 


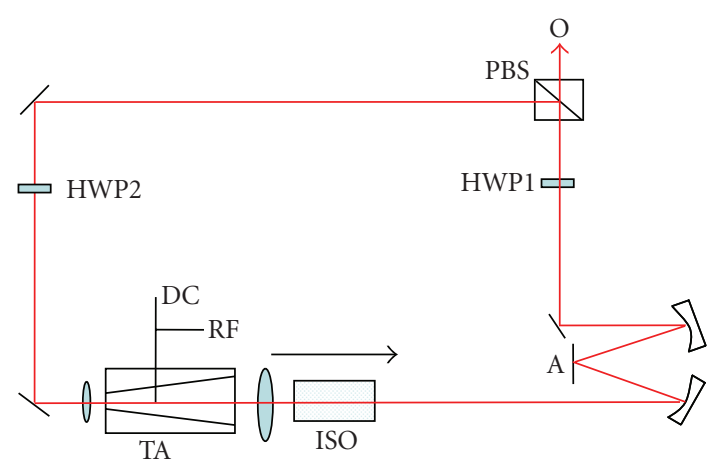

FIgURE 1: Schematic of hybrid mode-locked external ring cavity semiconductor laser. For mirror $A$ a normal mirror was used in $\mathrm{cw}$ configuration and a multiple quantum well mirror for modelocking. The radius of curvature of both focusing mirrors is $10 \mathrm{~cm}$.

tapered amplifier (Eagleyard, $780 \mathrm{~nm}, 1 \mathrm{~W}$, EYP-TPA-078001000-3006-CMT03-0000) is used as gain medium in a cavity. The advantages over the MOPA configuration are ease of alignment, a superior beam quality mostly defined by the cavity, and cost saving, since only one laser device is used. It is an unusual mode-locked oscillator for having the highest gain per cavity roundtrip as compared to most solid-state mode-locked oscillators. An additional benefit is the unanticipated short pulse duration. Subpicosecond pulses are generated by hybrid mode-locking without pulse compression. As compared to conventional mode-locked semiconductor lasers, the short pulse duration is achieved at a higher peak power and pulse energy.

\section{Experimental Results}

A commercially available TA is used, with focusing and collimating optics as well as an isolator. The optical isolator is required to protect the diode against any reflection into the large cross-section facet that may result in laser damage in the small cross-section input end of the device. For testing purposes, the TA was first characterized in an MOPA setup, to determine the input intensity (using a Ti:Sapphire laser as a source at $780 \mathrm{~nm}$ ) and pump current that lead to a saturated output. The maximum input power for which no gain saturation is observed, when increasing the pump current, was determined to be $7 \mathrm{~mW}$. At that input power, the maximum output power (at maximum pump current) is $610 \mathrm{~mW}$, corresponding to a small signal optical gain of 87 or $19.4 \mathrm{~dB}$.

A sketch of the ring cavity is presented in Figure 1, including the tapered amplifier, and a combination of a half wave plate (HWP1) and a polarizing beamsplitter (PBS) to limit the power coupled into the input end of the TA, while extracting the maximum amount of power from the ring laser. The second half wave plate (HWP2) adjusts the polarization to match the required input polarization of the TA.

In continuous wave operation (with a $100 \%$ reflection mirror at position $A$ in Figure 1), the output power (mea- sured at position $O$ in Figure 1) versus current characteristic of this laser shows a threshold current of $1.1 \mathrm{~A}$, and a spectrum centered at $781 \mathrm{~nm}$, with an FWHM of $0.58 \mathrm{~nm}$ at a temperature setting of $30^{\circ} \mathrm{C}\left(1.5^{\circ} \mathrm{C}\right.$ higher than the normal upper limit of the operational temperature range). The vertical and horizontal beam profiles shown in Figure 2 are seen to be nearly Gaussian with a $M^{2}$ of 1.07 . It appears thus that the spatial mode is determined by the external cavity, rather than by the tapered amplifier.

The relation between the TA input and the laser output (Figure 3) was measured by adjusting the output coupling via HWP1 and measuring the TA input through mirror M1. For this measurement, a multiple quantum well saturable absorber (MQW) is inserted in position $A$ of Figure 1. The peak output is well below the maximum possible output of the TA at the applied current and temperature due to the high losses in the cavity. Those losses result from lowreflectivity mirrors and the high absorption in the MQW. The measurement is not in the mode-locking operation, since the MQW modulation solely is not sufficient to start this mode.

Mode-locking is achieved by a combination of a multiple quantum well saturable absorber, and current modulation at the cavity repetition rate. Because of the very large gain of the amplifier, it is possible to use a large number of quantum wells, providing deep modulation. The MQW saturable absorber is designed with $10 \mathrm{Al}_{0.1} \mathrm{Ga}_{0.9}$ As layers-a composition that has been designed for saturable absorption at $795 \mathrm{~nm}$ [9] — deposited on top of a Bragg reflector. Double passage through this structure has a small signal absorption of $20 \%$ at the design wavelength. An RF signal at $88.2 \mathrm{MHz}$ from a standard signal generator is amplified (Mini Circuit ZHL-1-2W) to a peak-to-peak voltage of $4.5 \mathrm{~V}$, and applied via a bias-T of $50 \Omega$ impedance. The RF frequency is tuned to match the mode-spacing of the cavity. The mode-locked operation is remarkably less sensitive to the exact value of the RF frequency and the modulation depth, as standard actively mode-locked systems such as argon lasers, possibly because of the low quality factor of the empty cavity. An output power of $40 \mathrm{~mW}$ can be extracted from the polarizing beam splitter (output marked as $O$ in Figure 1). Higher power can readily be obtained since the TA can deliver $>500 \mathrm{~mW}$ output, but results in a degradation of the mode-locking. This is due to the fact that the higher gain is no longer matched by the nonlinear loss. It should be possible to solve this problem by increasing the modulation depth of the MQW, as has been done in other high gain mode-locked lasers, for instance, with 100 quantum wells [10]. Indeed, introducing an additional MQW of the same composition and $5 \%$ modulation leads to $60 \mathrm{~mW}(60 \%$ increase). In addition it led to the observation of self starting mode locking without RF modulation at low $(<10 \mathrm{~mW})$ output powers. The pump current had to be adjusted to $1.8 \mathrm{~A}$ to compensate for the additional losses. A salient feature of diode lasers is the short upper state lifetime and low saturation fluence, characteristics similar to that of dye lasers. As a result, there is no tendency to Q-switching as is the case in most solid-state lasers (e.g., Nd:vanadate, Ti:sapphire, and Cr:LiSAF,). The pure cw mode-locked regime is verified 


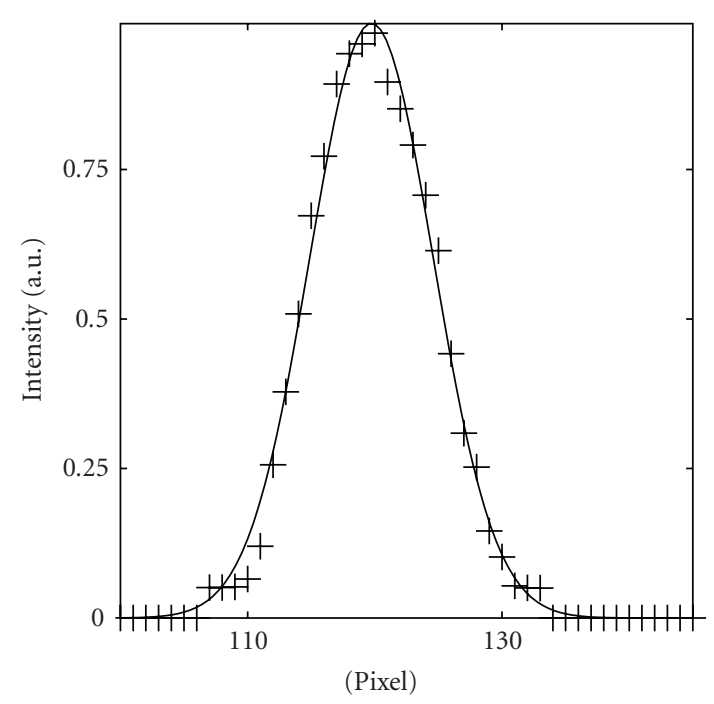

(a)

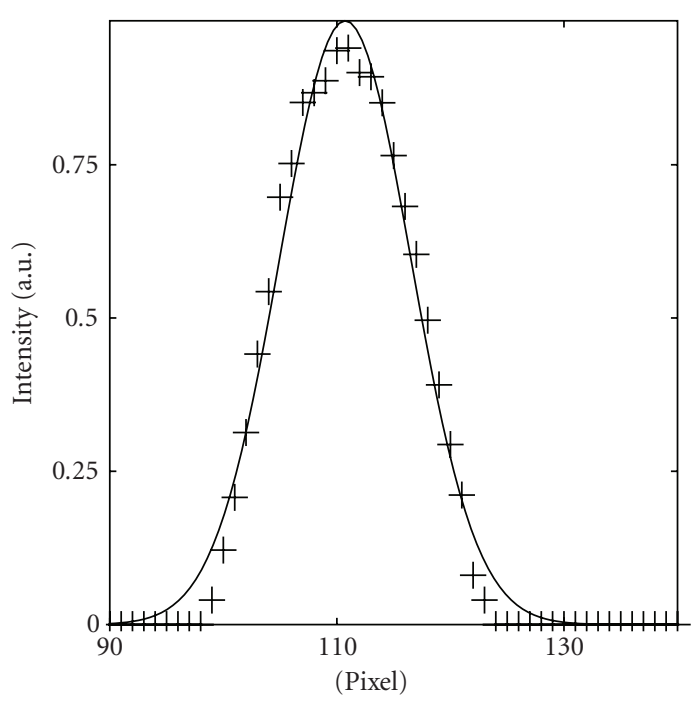

(b)

FIgURE 2: Laser-beam profiles with Gaussian fit in the horizontal (a) and vertical axis (b).

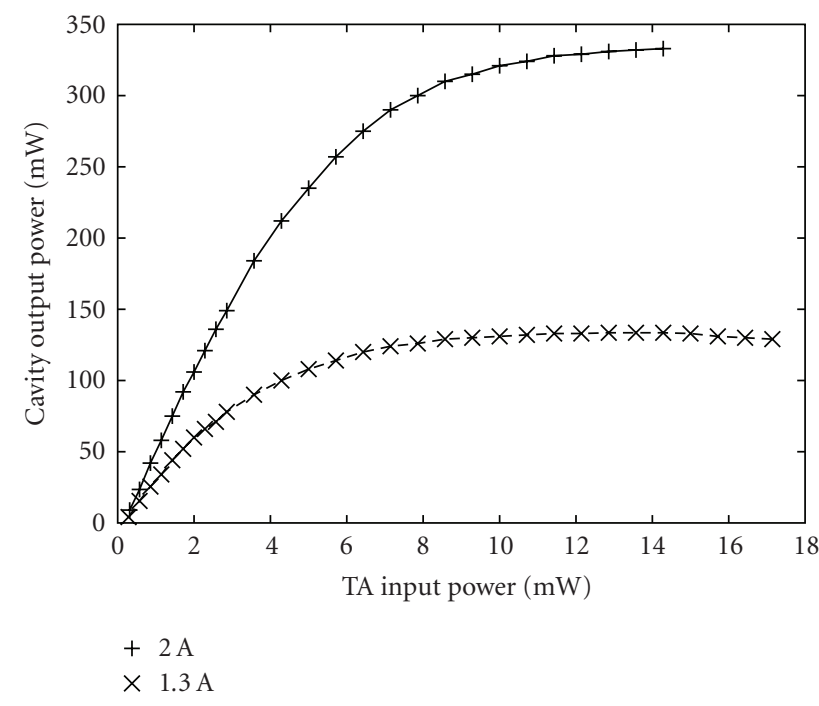

FIGURE 3: Laser output power versus intracavity TA input power at different pump currents (+) $2 \mathrm{~A}, \times 1.3 \mathrm{~A}$. The TA input power is monitored behind mirror M1.

with a detector-sampling oscilloscope combination (risetime $200 \mathrm{ps})$, an rf RF spectrum analyzer.

The pulses were further characterized by an optical spectrum analyzer (Ando AQ-6315A). The spectral width was measured to be $2.15 \mathrm{~nm}$ with the RF modulation signal applied, as compared to $0.58 \mathrm{~nm}$ without RF modulation (Figure 4).

A colinear autocorrelation with the exact ratio of $3: 1$ (Figure 5) indicates a well defined subpicosecond pulse [11]. The autocorrelation is seen to fit a $\operatorname{sech}^{2}$ shape of 0.5 psduration. duration. The observation of subpicosecond pulses from this laser was unexpected, given that there is no dispersion compensation. The short pulse duration can be

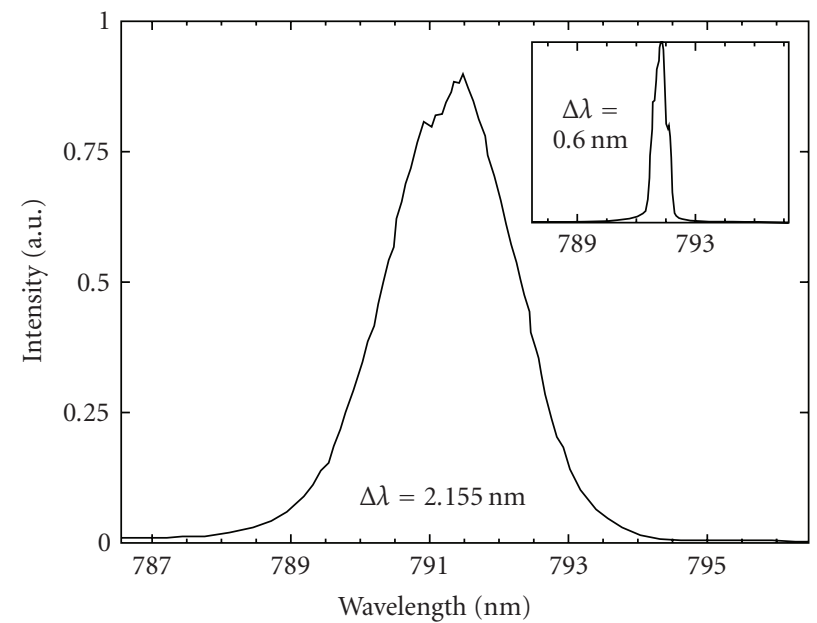

FIGURE 4: Optical spectrum with RF modulation on. Inset, RF modulation off.

explained by the large nonlinear attenuation (20\% to $30 \%$ ) of the MQW, as compared to the fraction of a percent used in conventional solid-state femtosecond lasers. The use of dispersion compensation should enable us to achieve shorter pulse durations since it will compensate to some degree the high self-phase modulation inside the semiconductor [12] and the dispersion from all the other cavity elements. Reduction of pulse duration by $30 \%$ in a semiconductor laser has previously been reported by using gratings [13] for dispersion management inside and outside the cavity. Since TA diodes are tunable over a range of $20 \mathrm{~nm}$, the spectrum can easily support shorter pulses. The repetition rate is limited by the bias-T to $100 \mathrm{MHz}$.

The pulse energy is limited by the comparably short lifetime of the excited electrons in the TA. It limits the amount of energy that can be stored in the TA at a given 


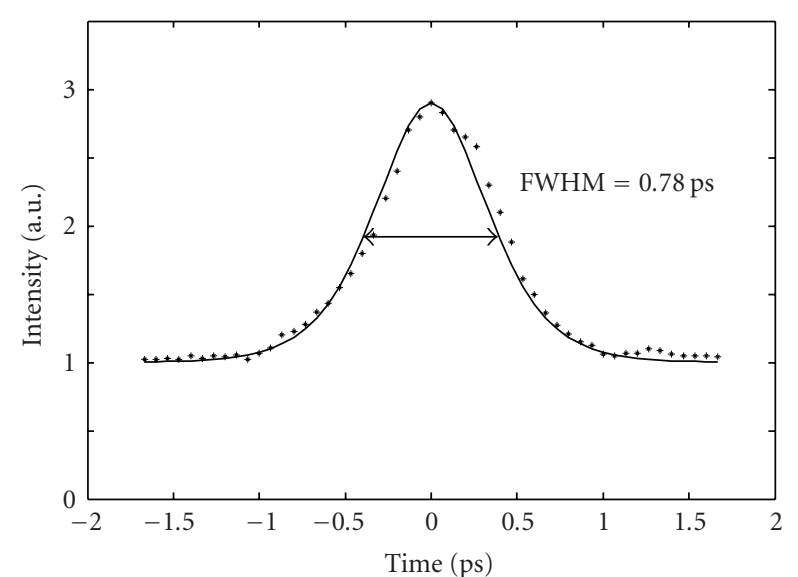

FIGURE 5: Intensity autocorrelation trace of generated pulse with pump current of $1.3 \mathrm{~A}$.

pump current. Since it is on the order of 1 nanosecond, decreasing the repetition rate below $1 \mathrm{GHz}$ will not result in an increase in pulse energy. A continuous wave output power of $1 \mathrm{~W}$ would thus lead to a maximum pulse energy of about $1 \mathrm{~nJ}$.

\section{Tunability of the Laser}

Two prism pairs inserted between the isolator and the MQW were used to test the tunability of the laser. Wavelength tuning is achieved by translating a $1 \mathrm{~mm}$ square aperture through the beam after the second cavity prism. Tuning curves shown in Figure 6 indicate a cw range of $20 \mathrm{~nm}$, while mode-locked operation is restricted to a $10 \mathrm{~nm}$ range. Very limited tuning (about $6 \mathrm{~nm}$ ) can additionally be achieved by adjusting the TAs operating temperature between $16^{\circ} \mathrm{C}$ and $28.5^{\circ} \mathrm{C}$. We were not able to get even close the desired wavelength of $795 \mathrm{~nm}$ within that temperature range set by the TA manufacturer. It should be noted that the wavelength range of the TA, when used to amplify a tunable master signal, is more than $40 \mathrm{~nm}$ according to the manufacturer specification, and significantly larger than what we observed from our cavity.

\section{Conclusion}

A novel external ring cavity hybrid mode-locked semiconductor laser, constructed with a tapered amplifier, is demonstrated. The high gain of the device makes it technically possible to introduce a large number of additional elements to improve its performance. Chirp compensation with gratings, prism, or coatings, and an MQW with a larger modulation depth are examples. Hybrid mode-locking is achieved by combining RF modulation with MQW saturable absorber, resulting in a minimum pulse length of 0.5 picosecond and an average power as high as $60 \mathrm{~mW}$. The peak power of $1.4 \mathrm{~kW}$, and total pulse energy above $0.68 \mathrm{~nJ}$ represent more than an order of magnitude improvement

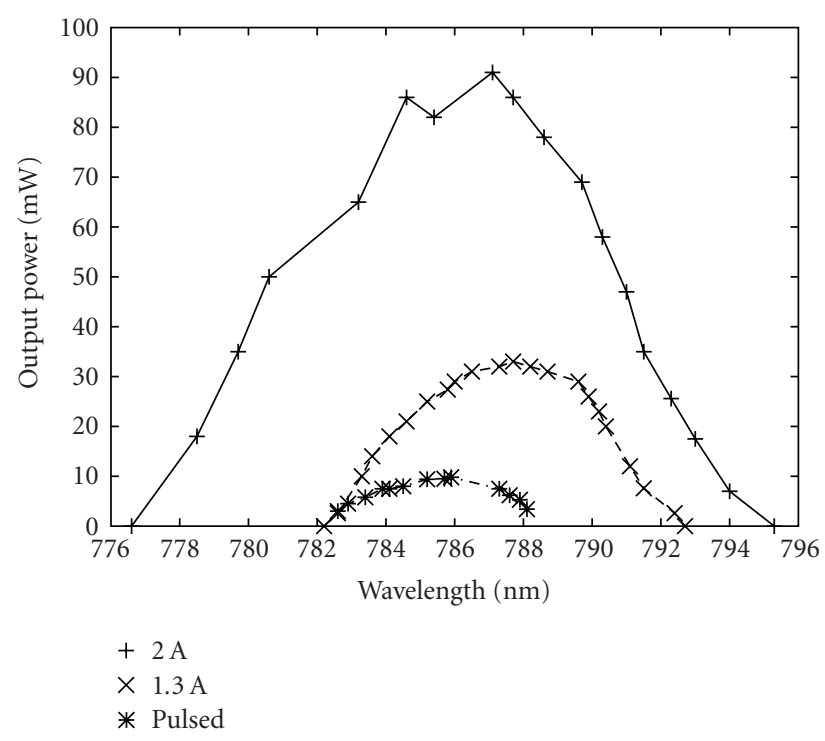

FIGURE 6: Output power versus wavelength for the cw laser at $2 \mathrm{~A}$ $(+), 1.3 \mathrm{~A}(\times)$, and for the mode-locked operation at 1.3 A. The TA temperature is set at $T=28.5^{\circ} \mathrm{C}$.

over those produced by conventional narrow stripe modelocked semiconductor lasers [13].

These properties, along with the stability, robustness, beam spatial quality, and ease to align, make this system a promising replacement for applications requiring high power $[14,15]$ and where measurements are performed intracavity [16]. The amplifier and MQW saturable absorber can be tailored to any wavelength in the visible-near IR range [9]. The high gain per round trip makes this an unusual and interesting mode-locked cavity. Further study, for example, of the effect of a change in the order of cavity elements could lead to a better understanding of mode-locked laser cavities in general.

\section{Acknowledgment}

This work was supported by NSF under Grant no. ECS0601612.

\section{References}

[1] Y. Silberberg and P. W. Smith, "Subpicosecond pulses from a mode-locked semiconductor laser," IEEE Journal of Quantum Electronics, vol. 22, no. 6, pp. 759-761, 1986.

[2] E. P. Ippen, D. J. Eilenberger, and R. W. Dixon, "Picosecond pulse generation by passive mode locking of diode lasers," Applied Physics Letters, vol. 37, no. 3, pp. 267-269, 1980.

[3] P. J. Delfyett, L. Flores, N. Stoffel, et al., "200-fs optical pulse generation and intracavity pulse evolution in a hybrid modelocked semiconductor diode-laser/amplifier system," Optics Letters, vol. 17, no. 9, pp. 670-672, 1992.

[4] A. Aschwanden, D. Lorenser, H. J. Unold, R. Paschotta, E. Gini, and U. Keller, "2.1-W picosecond passively mode-locked external-cavity semiconductor laser," Optics Letters, vol. 30, no. 3, pp. 272-274, 2005. 
[5] G. Bendelli, K. Komori, S. Arai, and Y. Suematsu, "A new structure for high-power TW-SLA," IEEE Photonics Technology Letters, vol. 3, no. 1, pp. 42-44, 1991.

[6] J. N. Walpole, E. S. Kintzer, S. R. Chinn, C. A. Wang, and L. J. Missaggia, "High-power strained-layer InGaAs/AIGaAs tapered traveling wave amplifier," Applied Physics Letters, vol. 61, no. 7, pp. 740-742, 1992.

[7] A. Mar, R. Helkey, J. Bowers, D. Mehuys, and D. Welch, "Mode-locked operation of a master oscillator power amplifier," IEEE Photonics Technology Letters, vol. 6, no. 9, pp. 10671069, 1994.

[8] Y. Xiong, S. Murphy, J. L. Caristen, and K. Repasky, "Design and characteristics of a tapered amplifier diode system by seeding with continuous-wave and mode-locked external cavity diode laser," Optical Engineering, vol. 45, no. 12, Article ID 124205, 5 pages, 2006.

[9] L. Arissian, J.-C. Diels, A. Stintz, and V. Kubecek, "Multiple quantum wells for ring and linear lasers with long lifetime gain," in Solid State Lasers XIV: Technology and Devices, S. Hoffman, Ed., vol. 5707 of Proceedings of SPIE, pp. 295-301, San Jose, Calif, USA, January 2005.

[10] W. Zendzian, J. K. Jabczynski, J. Kwiatkowski, et al., "Quasi cw laser diode side pumped Nd:YAG slab laser passively modelocked using multiple quantum well saturable absorbers," in Conference on Lasers and Electro-Optics (CLEO '07), p. 603, Optical Society of America, Baltimore, Md, USA, May 2007.

[11] J.-C. Diels, J. J. Fontaine, I. C. McMichael, and F. Simoni, "Control and measurement of ultrashort pulse shapes (in amplitude and phase) with femtosecond accuracy," Applied Optics, vol. 24, no. 9, pp. 1270-1282, 1985.

[12] E. Gehrig, D. Woll, M. A. Tremont, A. Robertson, R. Wallenstein, and O. Hess, "Saturation behavior and self-phase modulation of picosecond pulses in single-stripe and tapered semiconductor laser amplifiers," Journal of the Optical Society of America B, vol. 17, no. 8, pp. 1452-1456, 2000.

[13] B. Resan and P. J. Delfyett Jr., "Dispersion-managed breathingmode semiconductor mode-locked ring laser: experimental characterization and numerical simulations," IEEE Journal of Quantum Electronics, vol. 40, no. 3, pp. 214-221, 2004.

[14] S. Diddams, B. Atherton, and J.-C. Diels, "Frequency locking and unlocking in a femtosecond ring laser with application to intracavity phase measurements," Applied Physics B, vol. 63, no. 5, pp. 473-480, 1996.

[15] X. Meng, Y. Liu, and J.-C. Diels, "Femtosecond intracavity pumped ring optical parametric oscillator: an ultra-sensitive sensor," in Conference on Lasers and Electro-Optics (CLEO '04), San Francisco, Calif, USA, May 2004.

[16] J.-C. Diels, J. Jones, and L. Arissian, "Applications to sensors of extreme sensitivity," in Femtosecond Optical Frequency Comb: Principle, Operation and Applications, J. Ye and S. Cundiff, Eds., chapter 13, pp. 333-354, Springer, New York, NY, USA, 2005. 

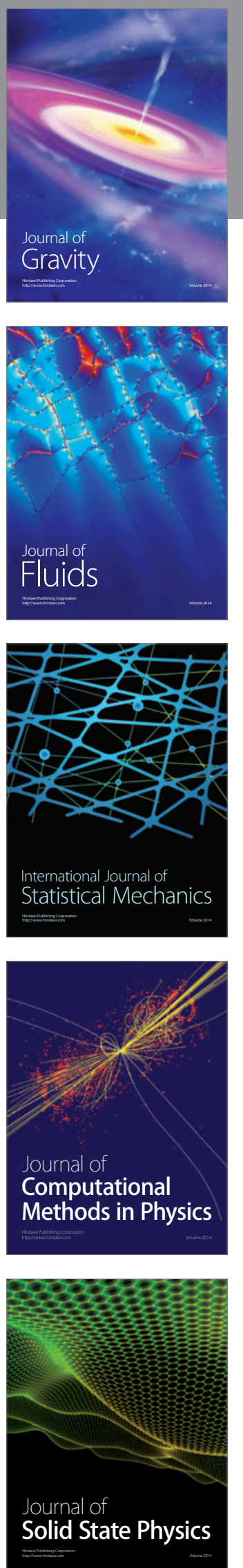

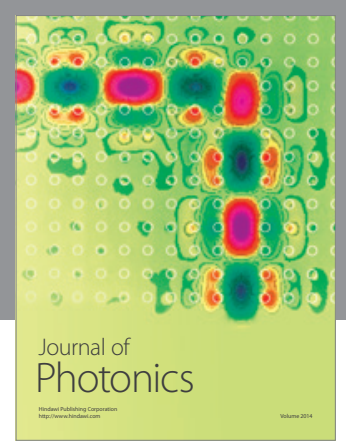

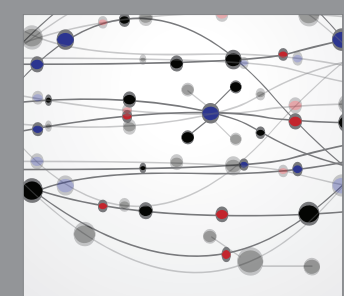

The Scientific World Journal
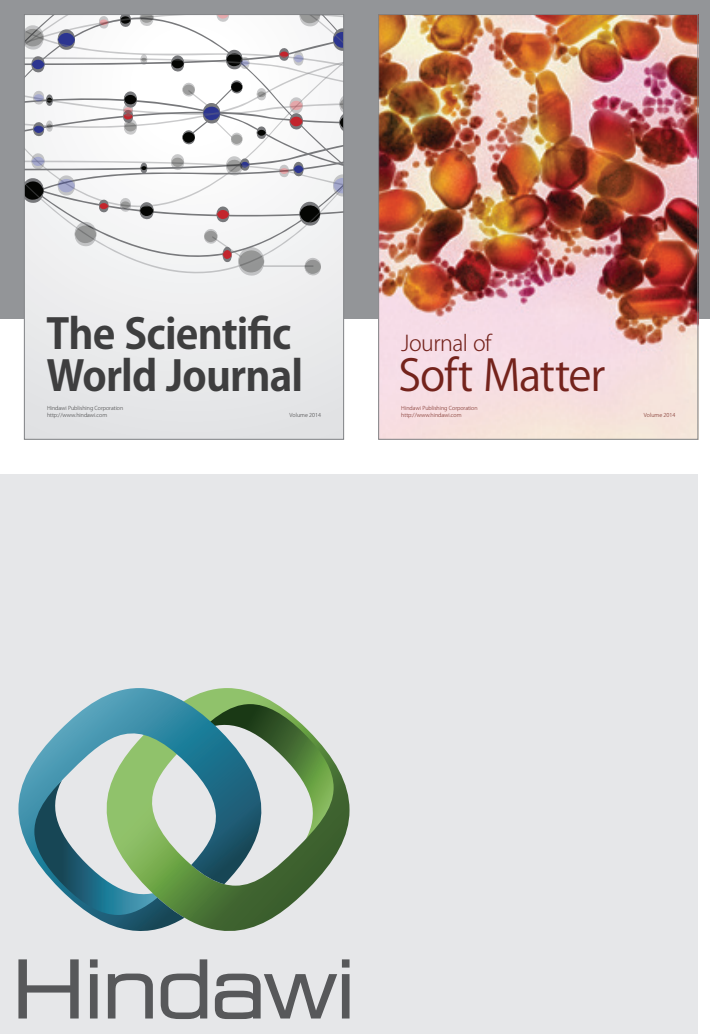

Submit your manuscripts at

http://www.hindawi.com
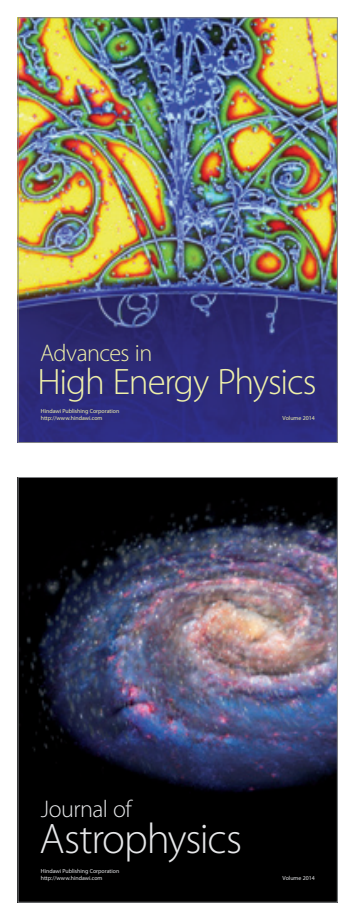
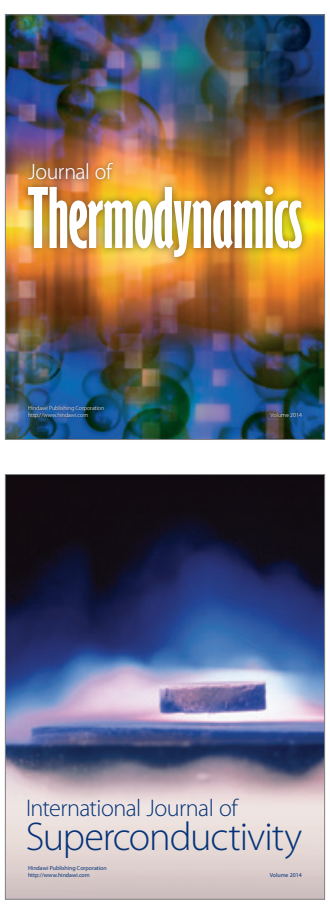
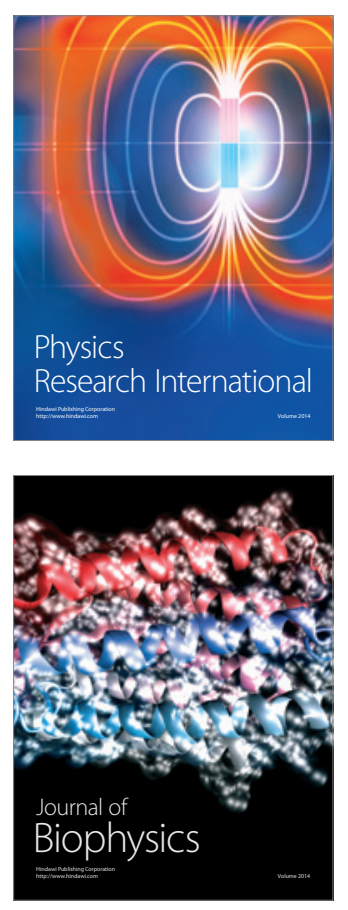
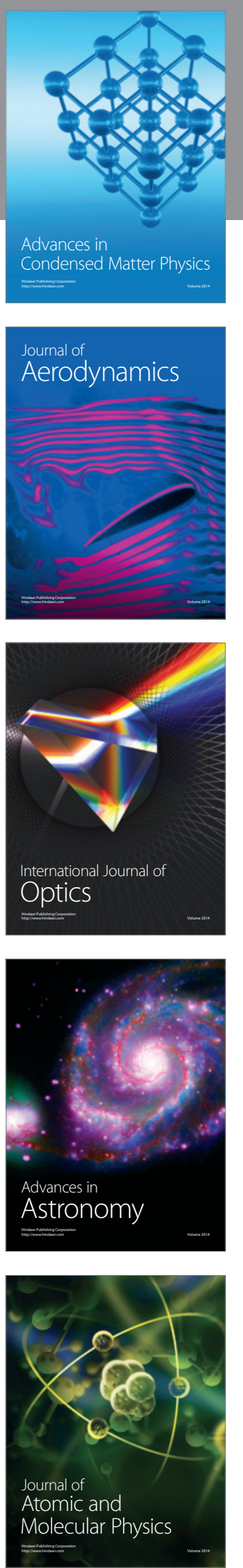